\title{
Exploiting Diverse Distance Metrics for Surrogate-Based Optimisation of Ordering Problems
}

\author{
Jim Smith \\ Department of Computer \\ Science and Creative \\ Technologies \\ University of the West of \\ England \\ Bristol, U.K. \\ james.smith@uwe.ac.uk
}

\author{
A Case Study
}

\author{
Christopher Stone \\ Department of Computer \\ Science and Creative \\ Technologies \\ University of the West of \\ England \\ Bristol, U.K.
}

\author{
Martin Serpell \\ Department of Computer \\ Science and Creative \\ Technologies \\ University of the West of \\ England \\ Bristol, U.K.
}

\begin{abstract}
Surrogate-assisted optimisation has proven success in the continuous domain, but only recently begun to be explored for other representations, in particular permutations. The use of Gaussian kernel-based models has been proposed, but only tested on small problems.

This case study considers much larger instances, in the experimental setting of a real-world ordering problem. We also investigate whether creating models using different distance metrics generates a diverse ensemble. Results demonstrate the following effects of use to other researchers: (i) Numerical instability in matrix inversion is a factor across all metrics, regardless of algorithm used. The likelihoood increases significantly once the models are parameterised using evolved solutions as well as the initial random population; (ii) This phase transition is also observed in different indicators of model quality. For example, predictive accuracy typically decreases once models start to include data from evolved samples. We explain this transition in terms of the distribution of samples and Gaussian kernel basis of the models; (iii) Measures of how well models predict rank-orderings are less affected; (iv) Benchmark comparisons show that using surrogate models decreases the number of evaluations required to find good solutions, without affecting quality.
\end{abstract}

\section{Keywords}

surrogate models; permutation representations; statistical disclosure control

\section{INTRODUCTION}

The landscape metaphor provides a natural way of visualising and analysing search problems. It occurs naturally

Permission to make digital or hard copies of all or part of this work for personal or classroom use is granted without fee provided that copies are not made or distributed for profit or commercial advantage and that copies bear this notice and the full citation on the first page. Copyrights for components of this work owned by others than ACM must be honored. Abstracting with credit is permitted. To copy otherwise, or republish, to post on servers or to redistribute to lists, requires prior specific permission and/or a fee. Request permissions from permissions@acm.org.

GECCO '16, July 20-24, 2016, Denver, CO, USA

(C) 2016 ACM. ISBN $978-1-4503-4206-3 / 16 / 07 \ldots \$ 15.00$

DOI: http://dx.doi.org/10.1145/2908812.2908854 when we impose a neighbourhood structure onto a set of candidate solutions. Thus, for a given instance of a problem, by imposing different neighbourhood structures we can create different landscapes, which may have different characteristics, . For many real-world problems fitness computation may be expensive (in terms of time, materials, or user attention). For such problems surrogate-assisted optimisation is commonly applied [1]. Figure 1 illustrates one common approach known as pre-selection.

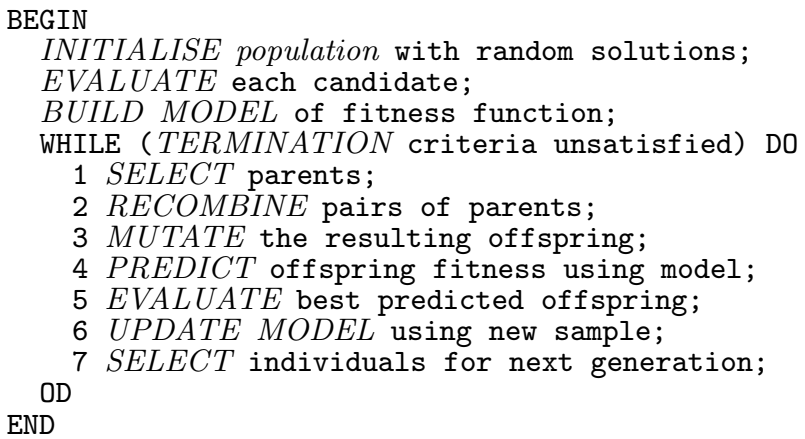

Figure 1: Surrogate-assisted optimisation using the pre-selection approach.

This paper considers surrogate-assisted optimisation of permutation problems from two perspectives:

- What is the effect of different distance metrics on the fidelity of the surrogate models learned from different problem instances?

- Are there practical details that need to be considered when applying these approaches to large permutationbased problems in an online optimisation context?

We conduct a case-study using a real-world optimisation problem from the field of Statistical Disclosure Control. Section 2 introduces relevant literature and concepts from surrogate optimisation and describes the Cell Suppression Problem. Section 3 describes our experimental methodology and 
Section 4 details the results obtained and their analysis. Finally, we draw conclusions from this study in Section 5 .

\section{BACKGROUND}

\subsection{Surrogate-Assisted Optimisation}

Surrogate models are widely used to assist in the optimisation of problems where the calculation of the fitness of candidate solutions is expensive - in time, materials, or other resources. Methods such as Kriging models and Efficient Global Optimisation [2] date back to the 1970s, and have been used on numerous occasions. In essence, a parameterised model of the underlying search landscape is fitted to a set of sampled solutions for which the "real" evaluation is available. Metaheuristic search is then carried out on this model landscape. Periodically, solutions are chosen for full evaluation, and the model is updated. Jin [3] has recently surveyed current issues in this area.

However, most work has been done in the continuous domain. It is only more recently that attention has been drawn to the combinatorial domain, first by Moraglio, Kim and coauthors $[4,5]$, and then by Zaefferer et al. [6, 7]. Moraglio and Kim [5] propose that the most appropriate approach for permutation landscapes is to build models using Radial Basis Function Networks (RBFNs). Given a set of $N$ sampled points, with fitnesses $f(i),(1 \leq i \leq N)$ a function (typically a Gaussian) is placed on each point. The estimated fitness of a permutation $x$ is given by the weighted sum:

$$
\tilde{f}(x)=\sum_{i=1}^{N} w_{i} \phi(i, x)
$$

where $w_{i}$ is the weight attached to the function centred on reference point $i$, and $\phi(i, x)$ is the radial basis function describing how the contribution to fitness changes with distance between the new solution $x$ and $i$. Typically in continuous domains the distance metric would be the Euclidean. Kim et al. have shown that this can be replaced by any choice of edit distance, and the RBFN gives a well-defined function. Thus in the case of Gaussian functions we get:

$$
\tilde{f}(x)=\sum_{i=1}^{N} w_{i} e^{-d \cdot \operatorname{dist}(i, x)^{2} / D_{\max }^{2}}
$$

where $D_{\max }$ is the maximum distance between any two permutations for a given distance metric. $d$ is a tunable "decay factor" (scaling element), whose value has been noted by Kim et al. to significantly affect results.

The model parameters $w_{i}$ are fitted to the data by minimising the difference between the values predicted by (1) and the observed values at the $N$ sample points. Let $\mathbf{A}$ denote a matrix with elements $A_{i j}=\phi(i, j) \forall i, j \in\{1, \ldots, N\}$, $\mathbf{w}$ the vector of weights $w_{i}$, and $\mathbf{f}$ the vector of initial sample fitnesses $f(i)$. If it is possible to find an induced model that perfectly matches the set of sample points, then in that case $\mathbf{A} \mathbf{w}=\mathbf{f}$ and we can solve this set of simultaneous linear equations to find the components of $\mathbf{w}$.

If $\mathbf{A}$ is positive definite, then its inverse will exist, and so the weights in the "ideal" model can be computed directly as $\mathbf{w}=\mathbf{A}^{-1} \mathbf{f}$. Matrix inversion is a complex, and often time-consuming task with many competing algorithms. Zaefferer et al. report some issues with numerical stability using Gauss-Jordan elimination [7], and suggest that Cholesky factorisation represents a more stable approach. Later, we will present some results examining this issue further and proposing a cause of the instability.

Assuming that the weight vector, (or a good approximation) can be found, the principal factor affecting the accuracy, or usefulness, of the model is the choice of the distance metric $d(i, x)$. Moraglio and Kim report on the use of Hamming and Swap distances [5], and Zaefferer et al. [7] investigate the use of 14 different metrics using a Kriging model, reporting significant differences in optimisation performance. The Kriging process creates a Maximum Likelihood Estimate (MLE) for each model during the modelbuilding process. Exploiting this, they propose a method that, given a set of samples, constructs the model for each distance metric and then selects for future use the one with the highest MLE. This could be seen as a crude form of an ensemble approach.

Here, we hypothesize that because each different distance metric will induce a different landscape, the relative distances between samples, or new data points whose fitness is to be estimated, will not be completely correlated. Therefore the errors in the estimation process may be uncorrelated, in which case combing their predictions to create an ensemble will provide a more accurate predictor [8].

\subsection{The Cell Suppression Problem}

\subsubsection{Description and Rationale for Case Study}

National Statistics Agencies (NSAs) publish useful statistical reports relating to their nation. However, they must ensure that the confidentiality of those who contributed the data to these reports is not compromised. When those tables contain magnitude data, the most common approach is Cell Suppression, where table cells that break confidentiality are suppressed (not published). These are referred to as primary suppressed cells $P$. Since these tables usually contain marginal totals, a subset of other "secondary" cells must also be chosen to be suppressed. The problem of selecting the secondary subset that protects the primary cells and minimises the information loss is known as the Cell Suppression Problem, and has been shown to be NP-Hard [9]. Therefore, in practice, published statistical tables are protected using a constructive approach. This creates a suppression pattern by considering the primary cells in sequence using an incremental linear programming (LP) heuristic model developed by Kelly et al. [9]. We have previously shown that the extent of over suppression is linked to the order in which the primary cells are considered [10], and so finding an approximate solution to the Cell Suppression Problem equates to finding the best permutation in which to protect the set $P$ of primary cells. We have also conducted benchmark experiments using local search and an Evolutionary Algorithm (EA) based on different mutation operators, and hence landscapes. These showed that the ranking of the quality of solution found on different landscapes, which reflects their "searchability", are highly instance-dependant, and that using a fixed ordering strategy is highly suboptimal [11].

The scale of the data published by NSAs is such that tables may typically contain millions of cells, tens, hundreds, or thousands of which may be sensitive, leading to very large sequences to be optimised. The presence of multiple dimensions and levels of hierarchy adds complexity to the LP. Although evolutionary approaches give state-of-the-art results 
in terms of solution quality and scalability [12], these come at the expense of time. To give an example, calculating the fitness of a single candidate solution (permutation of primary cells) on a representative four-dimensional table with around one million cells and only moderate levels of hierarchy can take more than 24 hours on a modern PC. Therefore we consider the Cell Suppression Problem represents an ideal case study for the use of surrogate-assisted optimisation.

\subsubsection{Problem Formulation}

Let $T$ denote a statistical table containing $n$ cells. The cell value $T_{i}, i \in\{1, \ldots, n\}$ is known to lie within the range $\left[l b_{i}, u b_{i}\right]$ and lower and upper protection limits $\left(l p l_{i}\right.$ and $\left.u p l_{i}\right)$ are provided by the National Statistics Agency. $z_{i}$ is the information loss associated with suppressing a cell $i$, and the cost to be minimised is $\sum_{i=1}^{n} c_{i}$, where $c_{i}=z_{i}$ if the cell is suppressed, and 0 if it is published.

The table's structure is captured via $m$ constraints, specified in a $n \times m$ matrix $M$, with $M_{i j}=-1$ if cell $i$ is the marginal total in constraint $j,+1$ if it is a component of the $j^{\text {th }}$ marginal sum and 0 otherwise. The variables $y_{i}^{-}$and $y_{i}^{+}$ are the lower and upper uncertainties provided by cell $i$.

For each primary cell $p$ in sequence two LPs are solved to protect the upper and lower protection levels. The first is:

$$
\min \sum_{i=1}^{n} c_{i}\left(y_{i}^{+}+y_{i}^{-}\right)
$$

subject to $M\left(y^{+}-y^{-}\right)=0,0 \leq y_{i}^{+} \leq U B_{i}$ and $0 \leq y_{i}^{-} \leq$ $L B_{i}$. The extra conditions for the upper protection levels are $y_{p}^{-}=0$ and $y_{p}^{+}=u p l_{p}-T_{p}$.

After solving this LP, any non-zero non-primary cell for which the LP has set $y_{i}^{-}+y_{i}^{+}>0$ is added to the suppression pattern and has its weighting $c_{i}$ set to 0 . An equivalent procedure is then carried out for the lower protection levels with the constraints $y_{p}^{-}=T_{p}-l p l_{p}$ and $y_{p}^{+}=0$.

\section{METHODOLOGY}

\subsection{Test Problems}

To aid comparison we used the test suite for which we have previously benchmarked our evolutionary approach against all the principal methods and reported improved performance [12]. Thus, if we can achieve benefits from using surrogate models within a similar algorithmic framework to that paper, we are improving on the state of the art. The test set contains 32 representative tables created by the UK Office for National Statistics and is publicly available $^{1}$. These vary in size from 1000 to 20,000 cells and have different dimensionality, levels of hierarchy, proportions of initially sensitive cells, and proportions of zero cells (which also influence instance hardness since they provide no benefit from suppression). The permutation problems range in size from length 50 to 1928 .

\subsection{Data Generation Methodology}

This paper concerns the practical implications of using surrogate models within a search context. Therefore, rather than consider large sets of randomly sampled points, we used a genetic algorithm (GA) to generate points in the search space, as these will have a different distribution - hopefully biased towards fitter solutions. Following as closely

\footnotetext{
$\overline{{ }^{1} \text { http://www.cems.uwe.ac.uk/ } \sim \text { jsmith/SDC_Webpage/ }}$
}

as possible [12], the GA, with a population size of 50, is initialised with two heuristically created orderings (decreasingcost and increasing-cost) plus 48 randomly generated permutations. Parent selection is by binary tournament, and a single offspring was created using order-based crossover (with probability 0.7 ), followed by mutation via the inversion of a randomly selected subsequence. The offspring replaces the worst in the population if fitter. For each test problem we ran 950 generations of a steady-state EA creating and evaluating one member per generation, thus creating 32 sets of 1000 samples.

From the resulting datasets we created RBF approximations and analysed their fidelity as a function of (i) the choice of distance metric, and (ii) the number of points $(N)$ used to create the models. In each case we kept the samples in the order they were created, using the first $N$ samples to create surrogate models, and the remaining $1000-N$ as the "test set" to measure fidelity and optimisation performance. To emphasize the point, the test set is never randomly distributed. If $N \leq 50$, (the initial population size) the training set will be randomly distributed, but thereafter some of the training set will come from a probability distribution created by the GA. The decision not to use cross validation was deliberate, since the premise of random sampling does not match actual run-time conditions if the optimisation process is successful.

For each combination of $N \in\{5,10,15, \ldots, 100\}$, "decay factor" $d \in\{1.0,2.5,5.0,10.0\}$, and problem instance, we ran the following process of creating models and making predictions for each of the (1000-N) test points:

- We created one RBF Network for each distance metric, and an ensemble predictor that averaged their outputs.

- We constructed a "dumb" classifier that made a constant prediction - the mean cost of the $N$ samples.

- In each case we also determined whether the matrix could be inverted by the Gauss-Jordan algorithm, or via Cholesky factorisation. We measured "success" as the case where $A A^{-1}=A^{-1} A=I$ to within a tolerance of $1 e^{-6}$, in which case we used this for creating the RBF Network.

- Otherwise we used Gaussian elimination to directly estimate a solution to the set of linear equations, and hence the weight vector.

\subsection{Comparison Metrics}

We considered fidelity in two ways. The first was absolute predictive accuracy, using the Mean Squared Error (MSE) over the test set. The second was whether a search algorithm that performed survivor selection using surrogate fitness would make similar decisions to one which had access to the full fitness information. This is a test of ranking accuracy rather than absolute value. For this we used two measures $\left(\rho^{\text {sel }}\right.$ and $\left.\rho^{\sim \text { sel }}\right)$ recommended by Jin [3],

To calculate these we picked $\lambda=100$ solutions from the test set and then ranked them according to the real and predicted fitness, before taking the top $\mu=20$ for each. $\rho^{\text {sel }}$ is calculated by counting how many individuals are selected by both methods and then normalising by the expectation for random selection. This gives a correlated value between -1 (using a surrogate model is worse than random) and +1 (selections from surrogate model match those from real fitness). $\rho^{\sim s e l}$ extends this analysis to consider the ranking 
within the selected individuals, which will be important if subsequent parent selection is fitness-based. In the calculation the true rank of each member selecting according to the predicted fitness is summed, then normalised as before. Full details of both measures may be found in [3]. This process was repeated 25 times for each problem instance and we report mean values below.

Finally, we ran comparison tests to examine the effectiveness and efficiency of the surrogate-assisted optimisation. Using the same initial populations as before, we re-ran the evolutionary algorithm with an added pre-selection phase in each iteration. 100 offspring were created and then evaluated by the surrogate model, then the one with the highest estimated fitness was re-evaluated using the full LP and considered for survivor selection as in the original algorithm. At each iteration the surrogate model was extended and re-parameterised to include the new information from the sample point.

\subsection{Surrogate Models}

In every case we used the set of $N$ training samples to create a radial basis function using a Gaussian kernel with width $d / D^{2}$ as per Eq. 1. To define the metrics, and the names used hereafter, consider two sequences $x$ and $y$ with $L$ elements that may have arbitrary labels:

- The Hamming distance, calculated as the number of positions in the sequence in which the elements occurring in the two solutions differed:

$\operatorname{Hamm}(x, y)=\sum_{i=1}^{L} \delta(x[i]-y[i])$,

where $\delta(k)=1$ if $k=0$, otherwise 0 .

- The Euclidean distance calculated as:

$\operatorname{Euc}(x, y)=\sqrt{\sum_{i=1}^{L}(x[i]-y[i])^{2}}$.

This metric considers cell id's, which could in theory be arbitrarily assigned, and so was initially expected to be meaningless. However, there is some natural structure to the ordering - tables are typically provided with "grand totals" as cell 0 , followed by a row or column totals, with the cells corresponding to individual categories typically last.

- The Position distance (Spearmann's footrule), is the summed differences in position for each element:

$\operatorname{Pos}(x, y)=\sum_{i=1}^{L}|i-j|$ where $y[j]=x[i]$.

- The Squared Position Distance (Spearmann's rank correlation co-efficient):

$\operatorname{Pos}^{2}(x, y)=\sum_{i=1}^{L}|i-j|^{2}$ where $y[j]=x[i]$.

- The $R$ metric [13], measures how many times an element immediately follows another in $x$ but not in $y$ (and vice-versa):

$R(x, y)=\sum_{i=1}^{L-1} \delta(x[i+1]-y[j+1])$ where $y[j]=x[i]$

- The Swap distance measures the distance on the swap landscape between two permutations, approximated by the number of pairs of elements for which the relative rankings differ in $x$ and $y$ :

$\operatorname{Swap}(x, y)=\sum_{i=1}^{L-1} \sum_{j=i+1}^{L} h(a-b)$

where $y[a]=x[i], y[b]=x[j]$ and $h(a)=1$ if $a>0$ otherwise 0 .
Since the theoretical maximum distances $D_{\max }$ are known, all distances are normalised to the range $[0,1]$ before use.

\section{RESULTS}

\subsection{Numerical Stability}

Figure 2 shows for each distance metric and decay factor, the proportion of matrices successfully inverted using the Gauss-Jordan algorithm (top) and via Cholesky factorisation (bottom) as $N$ increases. $N$ is the number of samples in the training set, or equivalently, the size of the RBF network (the surrogate model). On each plot a vertical line shows the point at which training set moves from the initial random population, to also containing evolved solutions.

In each case there is a clear transition for most metrics around $N \approx 50$ - most notably for Pos, SqPos, and Swap.

For the Gauss-Jordan approach, increasing the "decay factor", so that the contribution of each RBF diminishes more rapidly with difference, has little effect. In contrast, using Cholesky factorisation it has a more dramatic effect, making the process more stable, although still not sufficiently so that one could consider it a practical approach.

We hypothesize that the cause of the instability is that distances between evolved solutions and their parents tend to be closer than distances between the randomly created initial population. This would lead to small values in the matrix $A$ to be inverted. Given the length of the sequences considered, this could lead to numerical instability from division by very small numbers, which would be partially ameliorated by increasing the decay factor. Figure 3 shows this change in the minimum distance between samples in the training set as a function of its size $N$, and offers evidence to support this hypothesis.

We experimented with using a range of multipliers and logarithms in order to try and reduce the numerical instability, but these were only partially successful. To reduce the likelihood of coding errors or the impact of different implementations, we also tried three different implementations for each method taken from 'a mixture of academic sites and highly voted answers on stackexchange.com. This had little or no effect. By contrast, directly solving the system of simultaneous equations via Gaussian elimination, although marginally less accurate, proved notably more stable.

\subsection{Accuracy of Absolute Predicted Value}

The errors in the absolute predicted values were often considerable. Figure 4 illustrates this by plotting the number of test set samples predicted to have (impossible) negative cost as the training set size $(N)$ and the decay factor for the RBFs was varied. Although harder to measure, it seems like that a similar number may have had dramatically overpredicted costs. Figure 4 shows that:

- The number of samples incorrectly predicted to have negative costs rises with $N$ when the RBF network is trying to model the cost surface (landscape) for most distance metrics, especially for Pos and SqPos.

- Increasing the decay factor $d$, and hence the rate at which the contribution of each RBF falls off with distance, reduces the number of negative predictions in most cases.

- For $N<50, d=10$ there are no negative predictions on any problem instances with any distance metrics. 

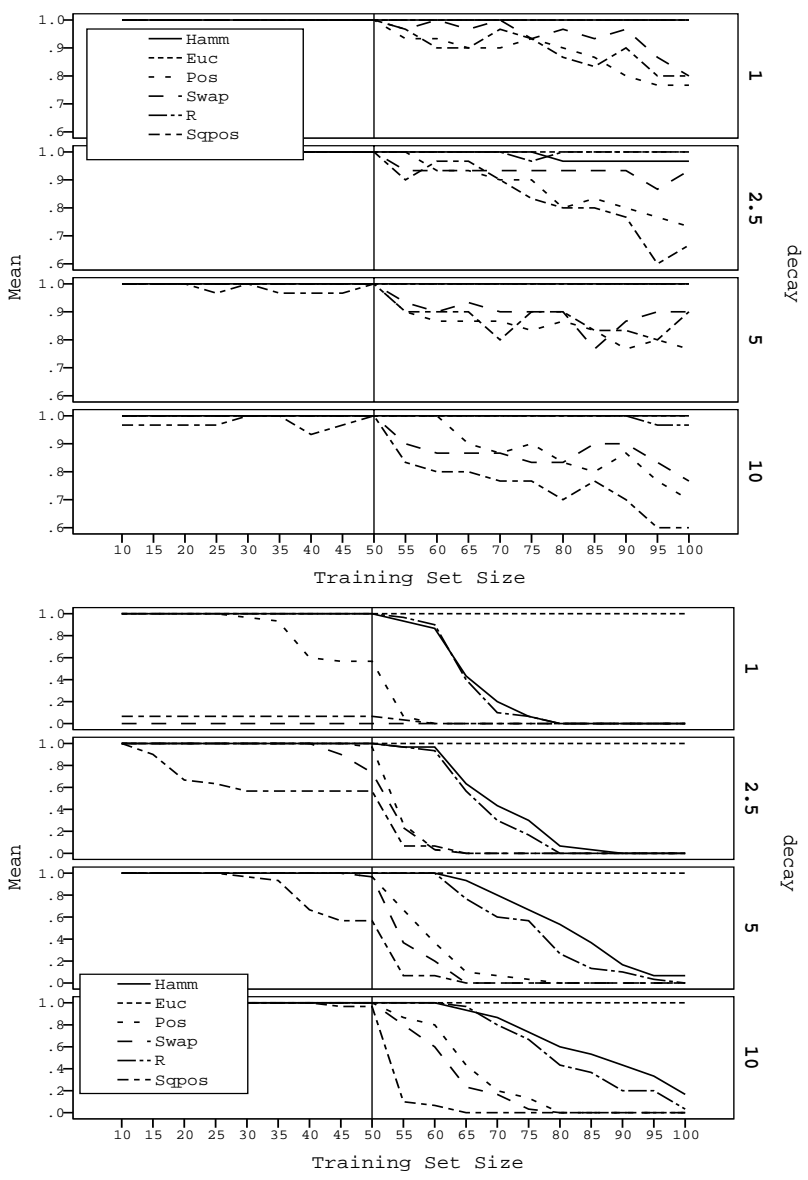

Figure 2: Proportion of matrices successfully inverted as a function of size $(N)$ and distance metric using Gauss-Jordan (top) and Cholesky (bottom) methods. Vertical reference line shows the point at which the training set contains evolved solutions as well as the initial randomised population.

A simple thought experiment illustrates a likely cause for the negative predictions. Imagine a single dimension with two closely spaced RBFs based on observed samples. If the samples have similar costs, then the surface can be modelled by assigning a nearly equal (roughly half-height) positive weight to each so that their summed contributions reach the desired costs at the known samples. Since both weights are positive, all points will have positive predicted costs. However, if the costs are very different, then the slope of the summed contributions needs to be sharply negative between them. The only way this can be achieved when both RBFs have large widths is if the weight of the RBF based on the higher-cost sample is large and positive, and the other is negative. Since the contributions of the RBFs to the summed cost prediction fall off with the square of the distance, this means that at some stage the prediction surface becomes negative. Moreover, the extent to which this happens will reflect the extent to which nearby pairs of samples have different costs - in other words the ruggedness of the landscape.

In practice, we curtailed the predictions to a lower bound of zero (since costs cannot be negative). Figure 5 shows the variation of mean MSE with the size of the RBF network

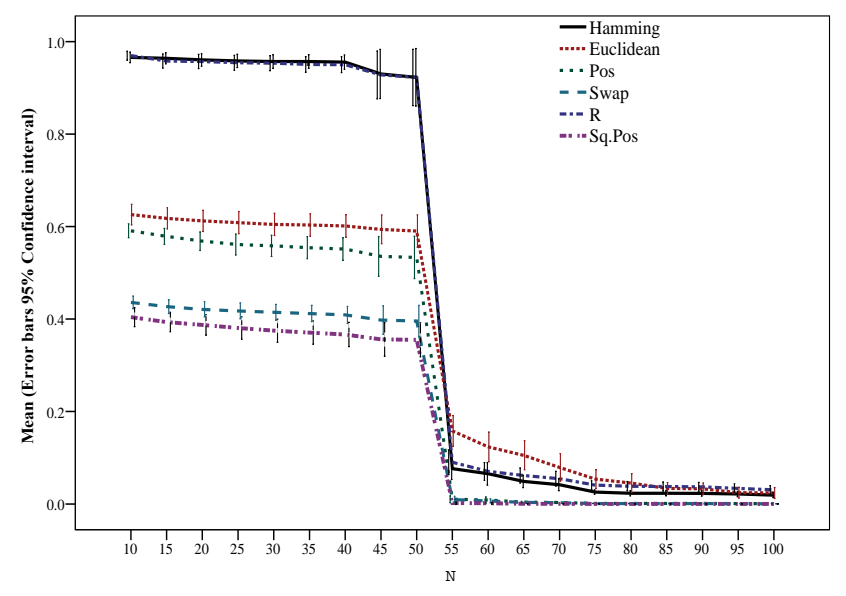

Figure 3: Change in minimum pairwise distance between samples in training set as a function of $N$. Plot shows mean (over all instances) value for each metric with error bars for $95 \%$ confidence intervals.

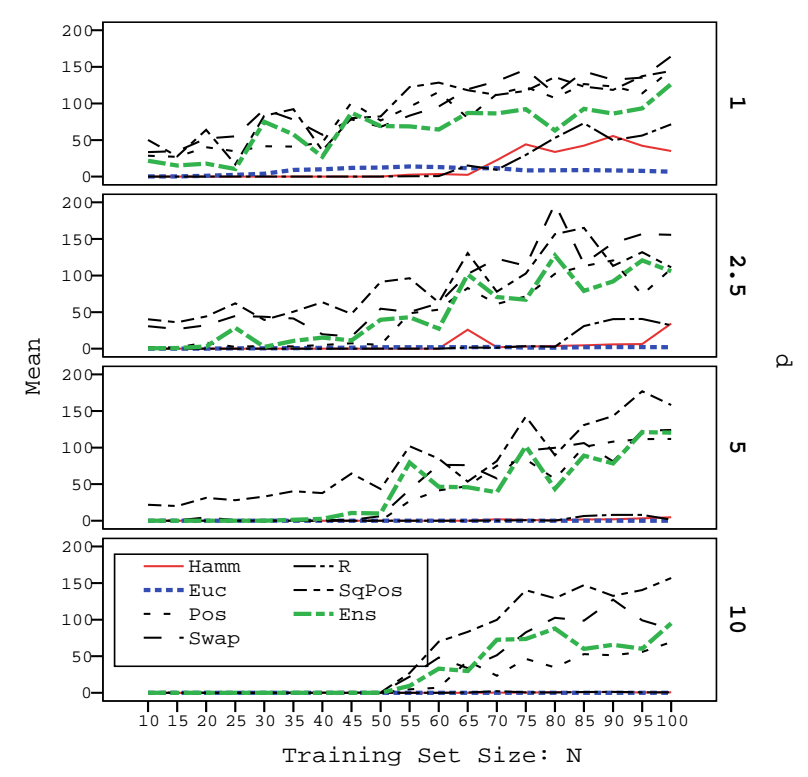

Figure 4: Line plot for each distance metric, and the ensemble, of the mean number of test samples predicted to have negative cost, as a function of $N$ and $d$. Maximum possible values are $1000-N$.

for different distance metrics, illustrated by the smallest, median, and largest, instances, which have lengths of 50, 517 and 1928 respectively. The same phase transition can be seen in the prediction accuracy as was noted in the numerical stability of the modelling process. To re-emphasise the implications of this point:

- At the start of a run of surrogate-assisted optimisation, when the models are built from a few random samples, the prediction error over the test set is relatively small.

- However, if the process involves rebuilding the model (which is standard practice), once the training set starts 
to include evolved solutions the observed prediction error actually increases.

Notably, the values of MSE are consistently higher for the problem instances with lower sequence lengths, hence smaller search spaces to be modelled. This may be because in the larger spaces more points are far from each other, and so the results are effectively regressing to the mean.

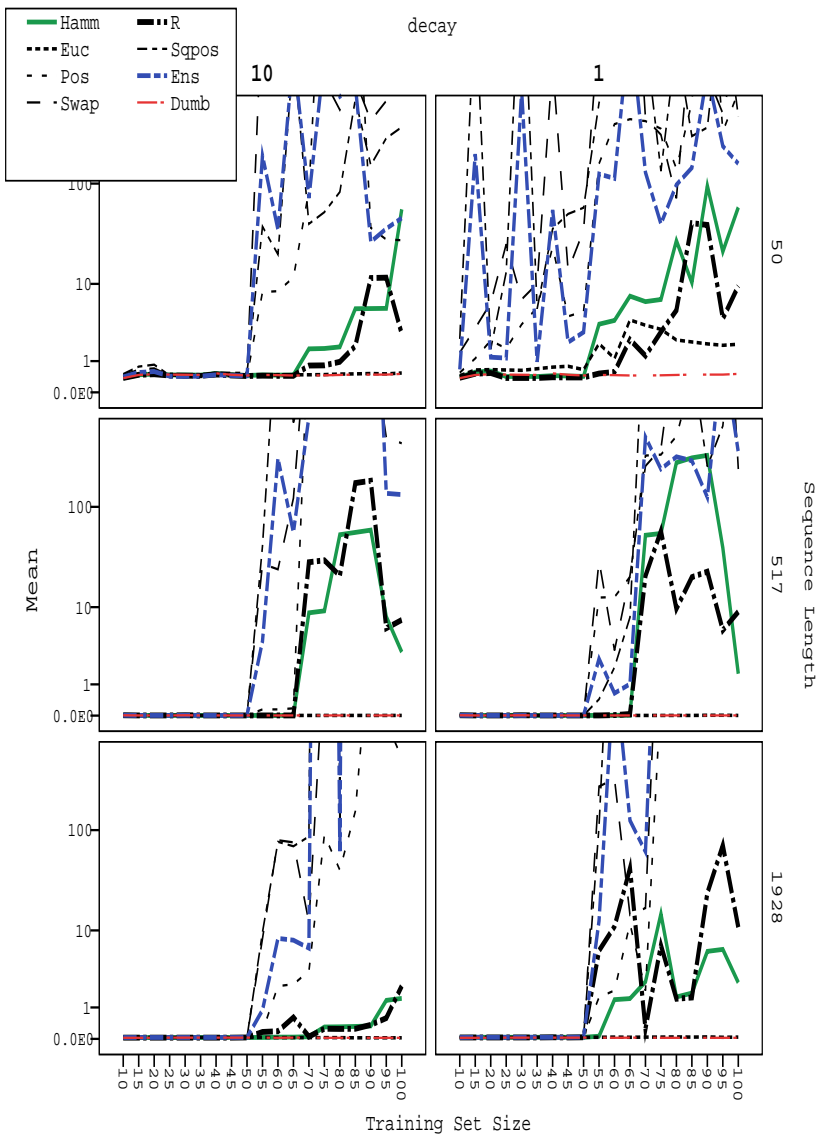

Figure 5: Three typical examples of how the absolute prediction error (MSE) changes with network size and decay factor for different distance metrics.

Further analysis shows that prior to the phase transition at $N=50$ there is little difference between the metrics. Space precludes full reporting of all the possible tests. Taking typical values of $d=1$ and $N=25$, Friedman's nonparametric test rejects the null hypothesis that the mean MSE for each metric is identical with more than $95 \%$ confidence. However, paired sample t-tests between the results for each metric and the "dumb" predictor show that the only significantly different group is the Swap metric - which has worse MSE results $(t=1.922, \mathrm{df}=29$, significance $=0.064)$.

For a typical value after the phase transition $(N=75)$, we see a similar pattern except that now it is the Pos metric which has significantly higher MSE values $(t=1.896, d f=29$, significance $=0.064$ ).

Taken at face value, these results suggest a risk that surrogate models are no more accurate at predicting absolute fitness values than a constant mean prediction, and often worse once evolved points are used for training. The next experiments investigate whether the surrogate models accurately predict solution ranking.

\subsection{Accuracy of Ranked Predicted Value}

Figures 6 and 7 illustrate the behaviour of $\rho^{\text {sel }}$ and $\rho^{\sim \text { sel }}$ as $d$ and $N$ are varied for three typical examples of different sizes. The phase transition effect is still present with these rank metrics, but much less marked than it was for MSE of absolute fitness.

Statistical analysis shows that the effect of $d$ is not significant for $\rho^{s e l}$ or $\rho^{\sim s e l}$. Although there is considerable variation with $N$, it is somewhat random in nature so a linear model has no predictive power. Averaged over all runs, both measures are positive for each distance metric. If an $\mathrm{RBF}$ Network had no predictive value, the value for each measure would be 0.0 , so we used a 1-sample Wilcoxon signed rank test to compare the median values against this constant. With 95\% confidence the null hypothesis (no difference) can be rejected for the Euc, Pos and R distance metrics with $\rho^{\text {sel }}$ and for all metrics with $\rho^{\sim s e l}$.

Overall these results suggest that although the estimated cost surfaces may be distorted relative to the true cost surfaces, they are preserving at least a partial rank-ordering of solutions, which is the critical measure for rank-based selection methods such as tournament or truncation selection in evolutionary algorithms.

\subsection{Optimisation Performance}

In the light of previous results, we compared the original GA results to those from an algorithm using a single surrogate model based on the Hamming distance (SMOHamm), and one using all six distance metrics and then taking an average to get an ensemble prediction (SMOEns).

In terms of effectiveness, the cost of the best solution found was lower for SMOHamm than for GA on 11 of the 32 instances, the same on 4 and higher on 17 . The mean difference was $0.034 \%$ and a paired samples t-test showed the effect was not statistically significant. In terms of efficiency, the time taken to find the best solution for that run was lower for SMOHamm than GA on 22 instances, the same on 2 and greater on 8 . Using a paired samples onetailed t-test showed that with more than $98 \%$ confidence we could reject the null hypotheses. Therefore we can conclude that using the Surrogate Model pre-selection phase statistically significantly decreased the number of solutions fully evaluated before the best solution was found.

A similar comparison of effectiveness and efficiency for SMOEns vs. GA yielded similar results. The the cost of the best solution found was the same on 4 instances and SMOEns and GA each found cheaper solutions on 14 instances. SMOEns found its best solution faster on 20 of the instances, slower on 8 of the instances and in the same time on 4 . The one-tailed paired samples t-test on speed between SMOEns and GA allowed us to reject the null hypothesis (no difference) with $96 \%$ confidence.

Analysis showed neither efficiency nor effectiveness differed significantly between the two surrogate-assisted algorithms.

\section{CONCLUSIONS}

This paper set out to show that surrogate-assisted optimisation using Radial Basis Function Networks was a valuable 


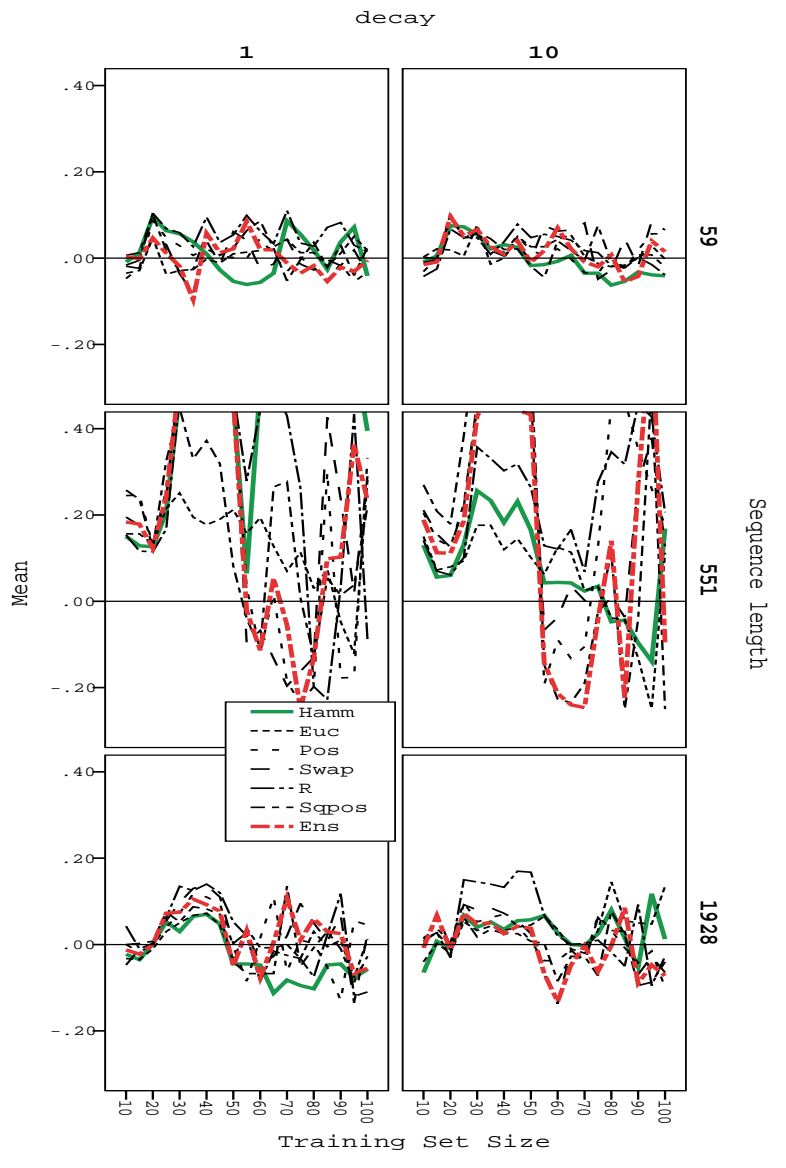

Figure 6: Examples of small, medium and large instances showing how the $\rho^{\text {sel }}$ measure of ranking predictions changes with network size and decay factor for different distance metrics.

approach for large combinatorial ordering problems with costly evaluation functions. We also hypothesized that producing several models using different distance metrics in the RBF kernels would naturally induce a diverse set of models, and so produce an ensemble with a low prediction error.

What we discovered in fact was rather different - although perhaps more useful as a source of guidance for practitioners:

- Numerical instability was a serious issue for the two most common methods of tuning the RBF weights, even when the matrices describing the linear system were positive definite. The observed occurrence of instability increased significantly once the algorithms started to included sample points create by evolution as well as the initial random population. Zaefferer et al. noted problems with the Gauss-Jordan approach [7]. Here, we observed even more significant problems with Cholesky decomposition. We have offered an explanation in terms of the increasing presence of samples very close to each other, which is supported by experimental evidence. Clearly, the message here is that when deploying RBF networks as surrogate models of permutation landscapes, it is necessary to check that the validity of the partial results involved in the

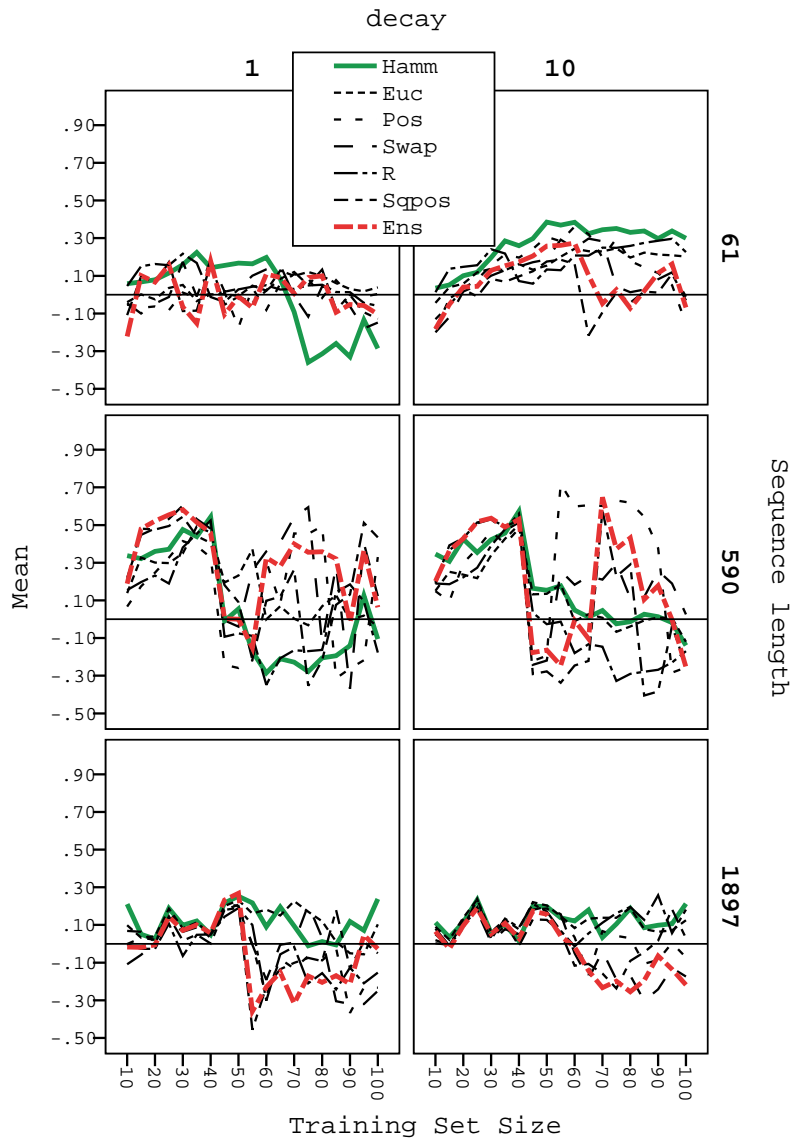

Figure 7: Examples of small, medium and large instances showing how the $\rho^{\sim s e l}$ measure of ranking predictions changes with network size and decay factor for different distance metrics.

process rather than assuming correctness, even with established code libraries. Our recommendation in the case of costly, or difficult to reproduce, fitness evaluations would be to employ several approaches in parallel, as is common practice in other mission-critical software design.

- Even when numerical stability was not an issue, we still observed an effect of increasingly poor prediction accuracy (for example, negative costs) in some cases. Again we offer an explanation in terms of the distribution of samples and the ruggedness of the landscape. We note that Kim et al. reported that the accuracy of their RBF models was significantly affected by the RBF widths [5], and we observed some similar affects. This suggests that possibly an automatic tuning procedure for $d$ could be devised by minimising the occurrence of negative predictions, and we will explore this in future work.

- Both of the effects above showed a distinct "phase transition" once the training set contained significant numbers of evolved solutions. This casts some doubt on the common, and intuitively sensible, practice of using all of the "true" fitness evaluations when model 
building. Although it might not seem wise to discard hard-earned information, our results suggest that it might be preferable to take into account proximity to other samples: either in selecting bases; or in locally tuning their widths rather than using a global value as previous authors have done.

- Given the above observations about occasional high error values, it is unsurprising that an ensemble of $\mathrm{RBF}$ networks using all of the distance metrics does not predict absolute cost values well. It remains for future work to see if a more selective approach to ensemble creation might pay dividends.

- The results for the measures comparing the rankings of solutions according to predicted or actual costs were more positive. This suggests that even given the effects and phase transitions noted above, the prediction landscapes do preserve at least partial rank ordering.

- In the context of evolutionary optimisation, results showed that although the surrogate-assisted optimisation did not statistically improve the best discovered solutions, it did allow those solutions to be found using significantly fewer calls to the true cost function. In other words, although the RBF Network model might not have sufficient fidelity to fine-tune search, they still contain enough information to allow the search process to more rapidly home-in on areas likely to contain good quality solutions.

In order to place these results in context, we return to the starting point of this paper: the successes of meta-heuristic search algorithms have lead to them being increasingly applied to more and more complex real-world problems with costly fitness evaluations. Surrogate-assisted optimisation has had many notable successes in the continuous domain, and current practice has been much influenced by experience gained from applications. A few recent papers on small scale "academic" problems have suggested that these benefits might be carried over to permutation representations, and this paper makes a contribution to the understanding needed to achieve those gains.

\section{ACKNOWLEDGMENTS}

The authors would like to thank officers of the UK Office for National Statistics for their insights and experience of Cell Suppression Problem.

\section{REFERENCES}

[1] Y. Jin. A comprehensive survey of fitness approximation in evolutionary computation. Soft Computing, 9(1): 3-12, 2005

[2] D. R. Jones, M. Schonlau, and W. J. Welch. Efficient global optimization of expensive black-box functions. Journal of Global Optimization, 13(4):455-492, 1998.
[3] Y. Jin. Surrogate-assisted evolutionary computation: Recent advances and future challenges. Swarm and Evolutionary Computation, 1(2):61-70, June 2011.

[4] A. Moraglio, Y. H. Kim, and Y. Yoon. Geometric surrogate-based optimisation for permutation-based problems. In Companion volume to the proceedings of GECCO 2011: the ACM-SIGEVO conference on Genetic and Evolutionary Computation, 2011.

[5] Y.-H. Kim, A. Moraglio, A. Kattan, and Y. Yoon. Geometric Generalisation of Surrogate Model-Based Optimisation to Combinatorial and Program Spaces. Mathematical Problems in Engineering, 2014(1):1-10, 2014.

[6] M. Zaefferer, J. Stork, M. Friese, and A. Fischbach. Efficient global optimization for combinatorial problems. In Proceedings of GECCO 2014, the 2014 Annual Conference on Genetic and Evolutionary Computation, pages 871-878. ACM Press, July 2014.

[7] M. Zaefferer, J. Stork, and T. Bartz-Beielstein. Distance measures for permutations in combinatorial efficient global optimization. In Proceedings of the 13th International Conference on Parallel Problem Solving from Nature. Springer, 2014.

[8] G. Brown, J. Wyatt, R. Harris, and X. Yao. Diversity creation methods: a survey and categorisation. Information Fusion, 2005.

[9] J. Kelly, B. Golden, and A. Assad. Cell suppression: Disclosure protection for sensitive tabular data. Networks, 22(4):397-417, 1992.

[10] A. Staggemeier, A. Clark, J. Smith, and J. Thompson. Improving our knowledge of metaheuristic approaches for the cell suppression problem. In Proceedings of Joint UNECE/Eurostat work session on statistical data confidentiality, December 2007.

[11] J. Smith, A. Clark, and A. Staggemeier. A genetic approach to statistical disclosure control. In Proceedings of GECCO 2009, the ACM-SIGEVO conference on Evolutionary Computation, pages 1625-1632. Springer, July 2009.

[12] J. Smith, A. Clark, A. Staggemeier, and M. Serpell. A genetic approach to statistical disclosure control. IEEE Transactions on Evolutionary Computation, 16(3):431-441, June 2012.

[13] V. Campos, M. Laguna, and R. Martõ. Context-independent scatter search and tabu search for permutation problems. INFORMS Journal on Computing, 17:11-122, 2005. 